\title{
Cutaneous mycology: a six-year experience in a tertiary hospital in Malaysia
}

\begin{abstract}
Introduction: Fungal infections of the skin is one the most common diseases encountered in dermatology. As a tertiary referral center in the state of Pahang, we sought to determine the epidemiology of patients with cutaneous fungal infections.

Methodology: A retrospective review of all biological specimens sent for fungal culture from the Department of Dermatology of Hospital Tengku Ampuan Afzan, Kuantan, and Pahang between 2011 to 2016 was carried out to determine the local pattern of cutaneous mycology.

Results: Out of 496 samples, $62.9 \%$ were nail clipping, $19.0 \%$ skin scraping, $12.9 \%$ hair and $5.2 \%$ skin biopsy tissue. Cultures were negative in 268 samples $(54.0 \%)$ and positive in $226(45.6 \%)$. Molds were the predominant fungus isolated $(74.0 \%)$, followed by yeasts $(14.7 \%)$ and dermatophytes $(11.3 \%)$. The predominant species isolated from hair and skin scraping samples was Microsporum canis (20/64 and 6/94 respectively), from nail samples Aspergillus niger (62/312) and from skin biopsy samples Sporothrix schenckii (3/26). In all four types of samples with positive cultures, the percentage of male and female subjects was almost equal.

Conclusion: More than half of all samples did not isolate any fungus despite a clinical diagnosis of cutaneous mycosis. This could be due to improper sampling methods without the aid of direct microscopy. Molds were the predominant fungi isolated from nail samples, whereas dermatophytes were the predominant fungi isolated from skin scraping and hair. Men and women were affected equally in all groups of samples with positive cultures.
\end{abstract}

Keywords: cutaneous mycoses, onychomycosis, dermatophytes, skin scraping, malaysia
Volume I Issue 4 - 2017

\author{
Rajalingam Ramalingam \\ Department of Dermatology, Hospital Tengku Ampuan Afzan, \\ Malaysia
}

\begin{abstract}
Correspondence: Rajalingam Ramalingam, Department of Dermatology, Hospital Tengku Ampuan Afzan, Malaysia, 25100 Kuantan, Email raj.blueheart@gmail.com
\end{abstract}

Received: October 27, 2017 | Published: December 01, 2017
Abbreviations: PCR, polymerase chain reaction; PAS, periodic acid-schiff; SPSS, statistical package for the social sciences

\section{Introduction}

A fungal infection of the skin is one the most common diseases encountered in dermatology. Cutaneous mycoses comprise superficial fungal infection of the skin, scalp and nails, as well as subcutaneous fungal infection. As a tertiary referral center in the state of Pahang, Malaysia, we receive many referrals for the further management of cutaneous mycoses, and as such, we sought to determine the clinic epidemiologic pattern of these patients.

\section{Methodology}

A retrospective review of all cutaneous specimens sent for fungal culture from the Department of Dermatology of Hospital Tengku Ampuan Afzan (HTAA), Kuantan, Pahang, Malaysia between 2011 to 2016 was carried out to determine the local pattern of cutaneous mycology. A comprehensive list of all cutaneous specimens sent from the Dermatology department to the Microbiology laboratory was obtained and the respective patient files traced and reviewed. Demographic data such as age, gender and ethnicity were recorded. Clinical data such as diagnosis and site of sampling was also noted. Microbiological data such as culture results were recorded. Data was subsequently analyzed using Statistical Package for the Social
Sciences (SPSS) version 22.0. In the microbiology laboratory, skin specimens were inoculated on Sabouraud's dextrose agar with and without $0.04 \%$ cycloheximide. All cultures were incubated at $30^{\circ} \mathrm{C}$ and checked twice weekly as per the laboratory protocol. A minimum of 4-6 weeks was allotted to confirm negative cultures.

\section{Results}

A total of 496 samples from 413 patients were sent, out of which $62.9 \%$ were nail clipping, $19.0 \%$ skin scraping, $12.9 \%$ hair and $5.2 \%$ skin biopsy tissue. Cultures were positive in 226 (45.6\%) samples from 193 patients. Overall, non-dermatophyte molds were the predominant fungus isolated $(74.0 \%)$, followed by yeasts $(14.7 \%)$ and dermatophytes (11.3\%). Positive cultures were obtained from $48.1 \%$ of nail clipping samples, $42.6 \%$ of skin scraping samples, $42.2 \%$ of hair samples and $34.6 \%$ of skin biopsy tissue samples. We found that there was scarce documentation of the anatomical site where samples were obtained from, especially with nail clipping and skin scraping samples (Figures $1 \& 2$ ). Positive fungal nail cultures were obtained from more women than men, while the other samples with positive isolates showed a slight male preponderance. The various demographic characteristics as well as the predominant fungal species isolated from each sample group are illustrated in (Table 1). Only three patients $(1.6 \%)$ had two concurrent types of cutaneous fungal infection; two with concurrent toenail onychomycosis and tinea pedis, and one with fingernail and toenail onychomycosis with tinea corporis. 
Table I Demographic Pattern of Cohort and Predominant Fungal Isolate from Various Groups of Samples

\begin{tabular}{|c|c|c|c|c|}
\hline Positive cultures & Nail & Skin scraping & Hair & Skin biopsy \\
\hline \multicolumn{5}{|l|}{ Patients } \\
\hline N & 135 & 31 & 19 & 8 \\
\hline Male: Female & $\mathrm{I}: 1.33$ & $1.21: 1$ & $1.7: 1$ & $1.67: 1$ \\
\hline Malay & $82(60.7 \%)$ & $24(77.4 \%)$ & $18(94.7 \%)$ & $5(62.5 \%)$ \\
\hline Chinese & $33(24.4 \%)$ & $5(16.1 \%)$ & 0 & $3(37.5 \%)$ \\
\hline Indian & $19(14.1 \%)$ & $2(6.4 \%)$ & 0 & 0 \\
\hline Indigenous & I (0.7\%) & 0 & I (5.3\%) & 0 \\
\hline Mean Age (Years) & $53.5(2-90)$ & $47.9(2-80)$ & $8.9(3-28)$ & $66.1(27-83)$ \\
\hline \multicolumn{5}{|l|}{ Samples } \\
\hline$N$ & 150 & 40 & 27 & 9 \\
\hline \multirow[t]{3}{*}{$\begin{array}{l}\text { Commonest Fungus } \\
\text { Isolated }\end{array}$} & A. niger: 62 (4I.3\%) & M. canis: 6 (15.0\%) & M. canis: 20 (74.I\%) & S. schenckii: 3 (33.3\%) \\
\hline & T. rubrum: 10 (6.7\%) & T. rubrum: 5 (I2.5\%) & M. audouinii: 5 (I8.5\%) & C. cladosporioides: I (I I.I\%) \\
\hline & Penicillium sp: 9 (6.0\%) & T. interdigitale: 3 (7.5\%) & E. floccosum: I (3.7\%) & Penicillium sp: I (1 I.1\%) \\
\hline
\end{tabular}

A. niger, Aspergillus niger; T. rubrum, Trichophyton rubrum; T. interdigitale, Trichophyton interdigitale; M. canis, Microsporum canis; M. audouinii, Microsporum audouinii; E. floccosum, Epidermophyton floccosum; S. schenckii, Sporothrix schenckii; C. cladosporioides, Cladosporum cladosporioides.

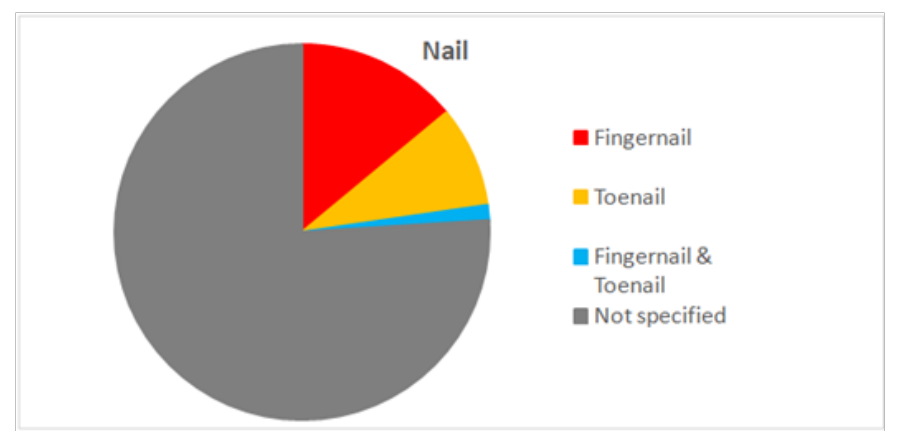

Figure I Type of Nail Clipping Samples.

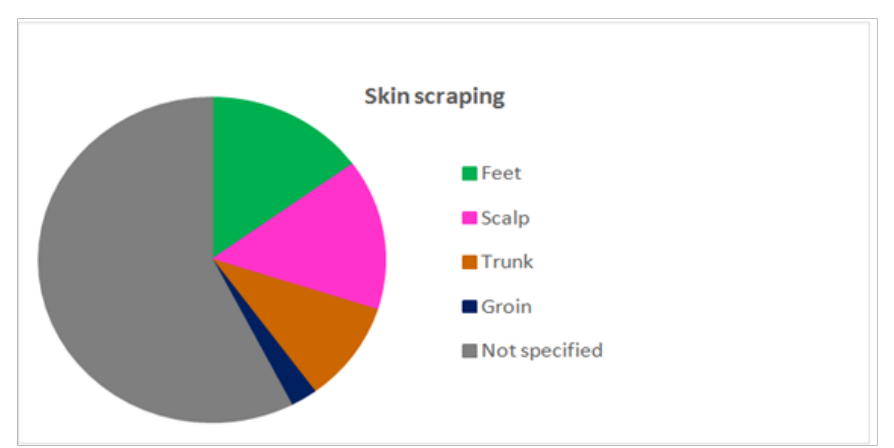

Figure 2 Site of Skin Scraping.

\section{Discussion}

More than half of all samples did not isolate any fungus despite a clinical diagnosis of cutaneous mycosis. This could be due to several possible factors, including a wrong diagnosis of cutaneous mycosis, a delay in specimen transportation to the laboratory, or less than proper sampling methods. It is recommended that specimens for fungal culture should be transported to the laboratory for immediate inoculation preferably within 2 hours of acquisition of a specimen, to ensure optimum recovery of fungi. ${ }^{1}$ Here, we stress the importance of good communication between the dermatology clinic and the receiving microbiology laboratory, especially prior to sending a specimen, in order to expedite specimen processing, thus ensuring the accuracy of culture results. Improper sampling methods may have also resulted in the high negative culture rate in our review. This may have been further aggravated by the lack of concomitant direct microscopy using potassium hydroxide $(\mathrm{KOH})$ or Wood's lamp examination. This is especially true in suspected cases of fungal nail infection, dermatophytosis and pityriasis versicolor.

For fungal nail infections, the recommended methods that results in a high culture yield are micro-drilling, proximal sampling and subungual curettage, ${ }^{2}$ as opposed to simple nail clipping. The quantity of the material obtained from the nail and the location from which the sample is taken greatly affects the culture result. The common practice in many local centres is distal nail clipping, which may result in a high prevalence of contaminants. The high prevalence of contamination can be effectively reduced by the simple practice of cleaning the entire nail plate, free edge of distal nail and hyponychium with an alcohol swab before clipping the distal end with a sterile nailclipper, and the nail specimen sent to the microbiology laboratory in either a sterile urine container or placed in a clean, folded piece of paper and labelled accordingly. While a micro-drill may not be readily available in most government outpatient clinics, simple curettage of the thickened subungual portion of the nail using a standard curette would still yield better results. If a curette is not available, scraping of the thickened nail plate with a no. 15 scalpel blade is also acceptable, with the nail scraping specimen sent to the laboratory using the methods described above. Interestingly, the direct microscopic examination of non-dermatophyte mold onychomycosis has been shown to yield a high false negative result of more than $42 \% .^{3}$ In addition, a recent meta-analysis concluded that the accuracy of $\mathrm{KOH}$ and cultures was lower compared to nail biopsy with Periodic AcidSchiff (PAS)-staining, ${ }^{4}$ something worth considering in patients with recalcitrant onychomycosis. 
For suspected tinea capitis, hair root and scalp skin scraping should be obtained by vigorously rubbing over the erythematous, scaly or alopecic region with a moistened cotton swab or gently rubbing with a sterile toothbrush. ${ }^{5}$ While this method certainly provides more accurate culture results, clinical features alone can point towards a diagnosis of tinea capitis, as demonstrated by one study where tinea capitis was confirmed by culture in $92 \%$ of children who had at least three of the following clinical features: scalp scaling, scalp pruritus, occipital adenopathy, and diffuse, patchy, or discrete alopecia. ${ }^{6}$ Apart from tinea capitis, epidermal scales from other types of dermatophytoses should be obtained by scraping the erythematous, peripheral, advancing edge of the lesion with a no. 15 scalpel blade after disinfecting the area with $70 \%$ alcohol. Scales should be instantly flaked into an open, sterile petri dish or sterile urine container to be transported immediately to the laboratory. When there is minimal scaling as with lesions over moist skin folds, it is preferable to use a strip of cellophane or vinyl tape to acquire the specimen. ${ }^{7}$ While skin biopsy with tissue culture remains the gold standard in diagnosing subcutaneous and deep fungal infection, occasional specimens can be difficult to culture either due to low fungal burden or heavy secondary infections, and may require multiple sampling, subcultures and/or extended incubation. ${ }^{8}$ Furthermore, there may be histopathologic identification of fungal elements despite skin tissue culture failing to show fungal growth. ${ }^{9}$ As such, future studies may want to look at the discrepancy rate between both methods of fungal identification.

\section{Recommendations}

We advocate proper prospective studies in the future addressing mycological patterns of cutaneous fungal infection with important, potentially associated factors such as comorbidities, immunosupression, occupation, social habits, socioeconomic status and chronic medication usage, as well as pets, hobbies, environmental factors and migration history. These associations if proven in future studies play a vital part in the management of cutaneous mycoses, such as avoidance of certain risk factors or modifications of specific lifestyle habits. Better sampling technique, cultures following direct $\mathrm{KOH}$ microscopy for a more accurate yield, as well as strict adherence to diagnostic criteria of cutaneous fungal infection in future audits will also no doubt aid in providing more accurate epidemiological data. The utilization of polymerase chain reaction (PCR) or matrixassisted lasers desorption/ionization time-of-flight mass spectrometry (MALDI-TOF) $)^{10}$ for a more accurate identification of fungal species should also be considered in future studies.

\section{Conclusion}

The predominant fungi isolated from nail samples in our review were non-dermatophyte molds, whereas samples of skin scraping and hair mainly isolated dermatophytes. Apart from onychomycosis, both superficial and subcutaneous mycoses of the skin and hair showed a male preponderance.

\section{Acknowledgements}

I would like to thank Dr Zulaiha A. Jalil and Dr Siti Khalijah M Zulkefli for their hard work in compiling the relevant records. There is no conflict of interest between myself, the author, and the aforementioned individuals. I would also like to thank the Director-General of Health, Malaysia, for his permission to publish this article.

\section{Conflict of interest}

The author declares no conflict of interest.

\section{References}

1. Chaya A K, Pande S. Methods of specimen collection for diagnosis of superficial and subcutaneous fungal infections. Indian $J$ Dermatol Venereol Leprol. 2007;73(3):202-205.

2. Shemer A, Trau H, Davidovici B, et al. Collection of fungi samples from nails: comparative study of curettage and drilling techniques. J Eur Acad Dermatol Venereol. 2008;22(2):182-185.

3. Bombace F, Iovene MR, Galdiero M, et al. Non-dermatophytic Onychomycosis Diagnostic Criteria: An Unresolved Question. Mycoses. 2016;59(9):558-565.

4. Velasquez Agudelo V, Cardona Arias JA. Meta-analysis of the utility of culture, biopsy, and direct $\mathrm{KOH}$ examination for the diagnosis of onychomycosis. BMC Infectious Diseases. 2017;17(166):2-11.

5. Friedlander SF, Pickering B, Cunningham BB, et al. Use of the Cotton Swab Method in Diagnosing Tinea Capitis. Pediatrics. 1999;104(2):276279.

6. Hubbard TW. The predictive value of symptoms in diagnosing childhood tinea capitis. Arch Pediatr Adolesc Med. 1999;153(11):1150-1153.

7. Miranda MF, Silva AJ. New uses of vinyl adhesive tape for reliable collection and diagnosis of common superficial mycosis. Skinmed. 2003;2(3):156-158.

8. Barros MB, de Almeida Paes R, Schubach AO. Sporothrix schenckii and Sporotrichosis. Clin Microbiol Rev. 2011;24(4):633-654.

9. Gonzalez Santiago TM, Pritt B, Gibson LE, et al. Diagnosis of deep cutaneous fungal infections: Correlation between skin tissue culture and histopathology. J Am Acad Dermatol. 2014;71(2):293-301.

10. Chalupová J, Raus M, Sedlárová M, et al. Identification of fungal microorganisms by MALDI-TOF mass spectrometry. Biotechnol Adv. 2013;32(1):230-241. 